\title{
TUMOUR OF THE BRAIN, ATROPHY OF RIGHT KIDNEY, WITH HYPERTROPHY OF THE LEFT KIDNEY.
}

\author{
Bx JOSEPH REDMOND, M.D., F.R.C.P.; \\ Physician to the Mater Misericordiæ Hospital; \\ Senior Physician to Cork-street Hospital.
}

[Read in the Section of Pathology, March 21, 1890.]

TrE specimens which I have the honour of exhibiting to-night before the Pathological Section were removed from the body of a patient, aged twenty-nine, who died in the Mater Misericordix Hospital on the 30th April, 1889. The details of the case may be of some interest on account of the large amount of attention that is being devoted at the present time to diseases and injuries of the brain. The notes of the case are as follows:-

CASE.-J. W., aged twenty-nine, a clerk by occupation, was admitted into the Mater Misericordiæ Hospital on the 29th April, 1889. His family history was as follows:- His father died of aneurysm, and a brother had recently died of peritonitis; his mother and other members of his family were living and healthy. He stated that he had been previously in the hospital under treatment for some urethral trouble, his stay in hospital on that occasion lasting from the 21st December to the 12th January. The patient was of temperate habits, and attributes his present illness to a blow which he received while attending a meeting towards the end of December, 1888. He stated that on that occasion he received a severe blow of a baton over the right ear, which, he said, did not completely stun him, but, as he described it himself, he was so much overpowered that it was with difficulty he succeeded in making his way home. From that time until his death he complained of severe pain in the right side of his head and suffered from fits, which usually occurred on Tuesday in each week. $\mathrm{He}$ attributed the regular recurrence of these fits on Tuesdays to the 
fact that Monday was always a busy day with him, his occupation being that of a railway clerk. When in a fit he says that he resembled a drunken man, so much so that on one occasion he was arrested by the police for being intoxicated, and confined in the cells for twelve hours. He states that he knows well when a fit is about to come on, and that during the attack, although he is apparently unconscious, yet he is quite well aware of all that is passing around him. The seizures, as observed in the hospital, were nut ushered in by any cry or moan-the patient simply sank into an apparently semi-conscious condition. There was no frothing at the mouth, nor was there any attempt at closing the teeth; there were no convulsive movements of the muscles of either the face or limbs; the pupils were normal, but slight pallor of the face was observed; when spoken to. the patient would not answer. These attacks lasted from about 10 to 15 minutes, and during this time the patient usually lay on his back, with his hands clasping the sides of his head, and from time to time would give vent to slow and deep moaning cries, as if he was suffering great pain. On the evening of his death he had several such seizures following one another in rapid succession, during one of which he expired, his pulse a few moments before death being 90 , heart action regular.

Post mortem (which was made by Dr. M'Weeney 52 hours after death)-Rigor mortis well marked. Body that of a well-nourished, muscular man.

On removing the skull cap the posterior part of the right parietal bone presented a very slight depression on its inner aspect, about the size of a shilling, surrounded by a slightly elevated reddish ring of bone. The dura mater was but loosely connected to the bone except at the above-mentioned spot, where it was rather closely adherent. The part of the membrane corresponding to the spot was opaque and white. On removing the dura mater it was found to be detachable from the pia mater with difficulty at this point, and this operation required the exercise of considerable care. The pia mater everywhere was deeply congested, and at the abovementioned point it was elevated slightly and white.

Brain remarkably soft. At the spot in question a tumour about the size of a pigeon's egg extended into the surface of the hemisphere. It was wedge-shaped, with thin edge inwards, and occu- 
pied the region of the angular and portion of the supra-marginal gyri. It was hard, and yellowish-white in colour. The surrounding brain substance had undergone white softening, so as to be almost diffluent. The rest of the brain seemed healthy enough. The ventricles, especially the left lateral, contained a considerable amount of serum.

Thorax-Both lungs were very deeply congested, but nowhere consolidated.

Heart-Healthy; firmly contracted.

Abdomen-Spleen slightly enlarged; very soft. Kidneys-Left kidney was very large and congested; ureter healthy. Right kidney was exceedingly small, so as to be scarcely recognised at first as such; the parenchymatous portion was smaller than the pelvis, which was distended with pale limpid fluid; only a few of the pyramids remained, and the glandular substance was nowhere one-fourth of an inch thick from pelvis to capsule. Right ureter was very mnch thickened, its walls greatly dilated, and appeared to be about the thickness of the little finger. Bladder apparently healthy. Liver abnormally adherent to the diaphragm, and a scar with a kind of puckered contraction was discovered on the diaphragmatic surface of the right lobe; on section it was very dark in coloured, and the vessels obviously very much congested.

Microscopic Examination of Tumour-The peripheral portion of the tumour consisted of small-celled structure, but its internal portion was structureless. It was surrounded by thick connective tissue, in which could be seen numerous small, thickened bloodvessels. The specimen under the microscope showed the disappearance of the nerve fibres from the cortex of the brain in the immediate neighbourhood of the tumour. The latter was probably of syphilitic character. 